\title{
ON AUTOMORPHISMS OF NODAL ENRIQUES SURFACES
}

\author{
BY F. COSSEC AND I. DOLGACHEV
}

1. Introduction. The purpose of this note is to announce a description of the automorphism group of a generic Enriques surface which contains a nonsingular rational curve (a nodal Enriques surface). The automorphism group of a generic unnodal Enriques surface was recently computed by W. Barth, C. Peters, and V. Nikulin (see [B-P]). As opposed to the transcendental methods of these authors, we work over any algebraically closed field of characteristic $p \neq 2,3,5,7$, and 17. Our notion of a generic nodal surface is very explicit: We assume that our surface belongs to an open Zariski subset of the 9-dimensional variety parametrizing Reye congruences of lines in $\mathbf{P}^{3}$. It is known that every generic nodal Enriques surface over the complex numbers is isomorphic to a Reye congruence [Co].

The main geometric ideas of this paper belong to A. Coble: We interpret differently and reconstruct his results on the number of the projective classes of quartic symmetroid surfaces congruent with respect to regular Cremona transformations [Cb 1, Cb 2].

2. Main results. Let $Q$ be the integral quadratic form of rank 10 defined by the Dynkin diagram of type $T_{2,4,6}$

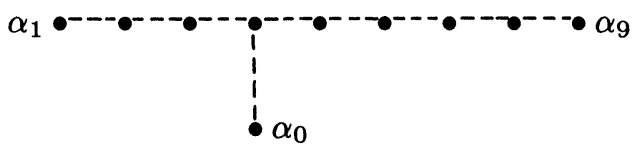

and let $W$ be the corresponding Weyl group generated by the reflections in the vectors $\alpha_{i}$ (see, for example, [Do]). It is known that $W$ contains a unique normal subgroup $\bar{W}(2)$ containing the 2-congruence subgroup $W(2)$ such that $W / \bar{W}(2)$ is isomorphic to the finite group $\operatorname{Sp}\left(8, \mathbf{F}_{2}\right)[\mathbf{G r}]$.

THEOREM 1. The automorphism group of a generic nodal Enriques surface is isomorphic to $\bar{W}(2)$.

Recall that this result is very similar to the cited result of Barth-Peters and Nikulin, where the answer is given in terms of the quadratic form defined by the Dynkin diagram of type $T_{2,3,7}$ (isomorphic to the Néron-Severi lattice of an Enriques surface).

THEOREM 2. The number of nonisomorphic nonspecial representations (resp. special) of a generic nodal Enriques as a double plane is equal to 34,780 (resp. 136).

We refer to $[\mathbf{A S}$, Chapter $\mathrm{X}]$ for the definition of special and nonspecial double plane representations of Enriques surfaces.

Received by the editors November 27, 1984.

1980 Mathematics Subject Classification. Primary 14J25.

(C)1985 American Mathematical Society $0273-0979 / 85 \$ 1.00+\$ .25$ per page 
THEOREM 3. Every two nonsingular rational curves on a generic nodal Enriques surface differ by an automorphism of the surface.

3. The group $\bar{W}(2)$. It is well known that the 2-congruence subgroup of the Weyl group of type $E_{7}$ or $E_{8}$ is generated by the unique element $\omega_{0}$ of maximal length. Our Dynkin diagram $T_{2,4,6}$ contains a subdiagram of type $E_{8}$ spanned by the vectors $\alpha_{0}, \alpha_{2}, \ldots, \alpha_{8}$ and two subdiagrams of type $E_{7}$ spanned by the vectors $\alpha_{0}, \alpha_{1}, \ldots, \alpha_{6}$ and $\alpha_{0}, \alpha_{2}, \ldots, \alpha_{7}$, respectively. Let $\omega_{0}^{(1)}, \omega_{0}^{(2)}$, and $\omega_{0}^{(3)}$ be the elements of $W$ defined by the corresponding elements $\omega_{0}$ associated to these subdiagrams. The group $\bar{W}(2)$ from the introduction contains the elements $B=\omega_{0}^{(1)}, K=\omega_{0}^{(2)}, G=\omega_{0}^{(3)} s_{9}$, where $s_{9}$ is the reflection in $\alpha_{9}$.

Proposition 1. (i) The group $W(2)$ is the smallest normal subgroup of $W$ containing the elements $B$ and $K$.

(ii) The group $\bar{W}(2)$ is the smallest normal subgroup containing the elements $B, K$, and $G$.

The first assertion was stated without proof by Coble $[\mathbf{C b} 2]$. A similar statement for the Weyl group of type $T_{2,3,7}$ was also stated by him and has been proven recently by E. Looijenga (unpublished). Our proof follows the idea of Looijenga. The second assertion follows easily from the first one.

4. Reye congruences. It is known that every Enriques surface $F$ can be embedded into $\mathbf{P}^{5}$ as a surface of degree $10[\mathbf{C o}]$. Also it is known that a nodal Enriques surface must contain a nonsingular rational curve of degree at most 4 [C-D].

Proposition 2. Let $F$ be a nodal Enriques surface of degree 10 in $\mathbf{P}^{5}$. Assume that $F$ does not contain nonsingular rational curves of degree $<4$. Then $F$, or its reembedding by $\left|O_{F}\left(K_{F}(1)\right)\right|$, is isomorphic to a Reye congruence embedded into $\mathbf{P}^{5}$ by Plücker coordinates.

Recall that a Reye congruence is defined as the variety $R(T)$ of lines in $\mathbf{P}^{3}$ contained in at least two quadrics from a general web $T$ of quadrics in $\mathbf{P}^{3}$. Clearly, Proposition 2 gives a satisfactory geometric notion of a generic nodal Enriques surface.

5. Sketch of the proofs. Let $R(T)$ be a Reye congruence, and let $H(T)$ be the Hessian surface of $T$, the quartic symmetroid surface in $\mathbf{P}^{3}$ parametrizing the singular quadrics from $T$. We assume also that $R(T)$ does not contain nonsingular rational curves of degree $\leq 3$ (cf. Proposition 2). Then $H(T)$ possesses ten distinct nodes corresponding to the quadrics of rank 2 from $T$. Moreover, there are no discriminant conditions on these ten nodes in $\mathbf{P}^{3}$ (see [Do]). The projective isomorphism classes of ordered ten nodes of such quartic symmetroids are represented by an algebraic variety $V$. It is known that a minimal nonsingular model $S(T)$ of $H(T)$ is isomorphic to the $K 3$ cover of $R(T)$. Let $q$ be the corresponding involution on $S(T)$. The Weyl group $W$ acts regularly on $V$ via its Coble representation [Do]. The kernel of this representation contains the elements $K, B$, and $G$, which correspond to 
the Kantor, dilated Bertini, and dilated Geiser birational involutions in $\mathbf{P}^{3}$, respectively (see $[\mathbf{C b}$ 2]). By Proposition 1 it contains the whole subgroup $\bar{W}(2)$. Since $W / \bar{W}(2)=\operatorname{Sp}\left(8, \mathbf{F}_{2}\right)$ is a simple group, one can easily see that the kernel of the Coble representation is exactly $\bar{W}(2)$. Thus, the finite group $W / \bar{W}(2)$ acts on $V$, and there exists an open Zariski subset $V^{\prime}$, where it acts without fixed points $\left(\operatorname{char}(k) \nmid \# \operatorname{Sp}\left(8, \mathbf{F}_{2}\right)\right)$. However, the isotropy subgroup $W_{v}$ of a point $v \in V$ can be identified with the automorphism group of the variety $X_{v}$ obtained by blowing up the corresponding ten points in $\mathbf{P}^{3}[\mathbf{D o}]$. Next, we notice that the symmetroid surface $S(T)$ lies in $X_{v}$ and represents the isolated $\left|-2 K_{X}\right|$. We prove that $W_{v}$ can be identified with the group of automorphisms of the corresponding $K 3$ surface $S(T)$ which commute with the involution $q$ (modulo the involution itself): i.e., with the automorphism group of the Enriques surface $R(T)$. This finishes the proof of Theorem 1 . The other two theorems follow from the first one after proving that the group $W$ acts on the set of polarizations of degree 2 on $F$ (resp., nonsingular rational curves on $F$ ) and computing the orbits of this action and the corresponding stabilizer groups.

\section{REFERENCES}

[AS] I. Shafarevich (Editor), Algebraic surfaces, Proc. Steklov Math. Inst., vol. 75, Moscow, 1964.

[B-P] W. Barth and C. Peters, Automorphisms of Enriques surfaces, Invent. Math. 73 (1983), 383-411.

[Cb 1] A. Coble, Algebraic geometry and theta functions, Amer. Math. Soc. Colloq. Publ., vol. 10, 3rd ed., Amer. Math. Soc., Providence, R.I., 1968.

[Cb 2] _ The ten nodes of the rational sextic and of the Cayley symmetroid, Amer. J. Math. 41 (1919), 243-265.

[C-D] F. Cossec and I. Dolgachev, Smooth rational curves on Enriques surfaces, Math. Ann. (to appear).

[Co] F. Cossec, On the Picard group of Enriques surfaces, Math. Ann. (to appear).

[Do] I. Dolgachev, Weyl groups and Cremona transformations, Proc. Sympos. Pure Math., vol. 40, Part 1, Amer. Math. Soc., Providence, R.I., 1983, pp. 283-294.

[Gr] R. Griess, Jr., Quotients of infinite reflection groups, Math. Ann. 263 (1983), 267-278.

Department of MATHEMAtics, University of Michigan, AnN ARbor, MICHIGAN 48109 